\title{
Follow-up care by a genetic counsellor for relatives at risk for cardiomyopathies is cost-saving and well-appreciated: a randomised comparison
}

\author{
Karin Nieuwhof ${ }^{\star, 1}$, Erwin Birnie ${ }^{1}$, Maarten P van den Berg ${ }^{2}$, Rudolf A de Boer ${ }^{2}$, Paul L van Haelst ${ }^{3}$, \\ J Peter van Tintelen ${ }^{4}$ and Irene $M$ van Langen ${ }^{1}$
}

Increasing numbers of patient relatives at risk of developing dilated or hypertrophic cardiomyopathy (DCM/HCM) are being identified and followed up by cardiologists according to the ACC/ESC guidelines. However, given limited healthcare resources, good-quality low-cost alternative approaches are needed. Therefore, we have compared conventional follow-up by a cardiologist with that provided at a cardiogenetic clinic (CGC) led by a genetic counsellor. Phenotype-negative first-degree relatives at risk for DCM/HCM were randomly assigned to see either a cardiologist or to attend a CGC. Uptake and resource use were recorded. For 189 participants, we evaluated quality of care experienced, patient satisfaction and perceived personal control (PPC) using validated questionnaires and estimated the average cost difference of these two modes of care. Maximum patient satisfaction scores were achieved more frequently at the CGC $(86 \%$ vs $45 \%, P<0.01)$. In terms of follow-up care provided, the genetic counsellor did not perform worse than the cardiologist $(95 \%$ vs $59 \%, P<0.01)$. The genetic counsellor more often enquired about the relative-at risk's health (100\% vs $65 \%, P<0.01)$ and family health $(97 \%$ vs $33 \%, P<0.01)$, measured blood pressure $(98 \%$ vs $29 \%, P<0.01)$ and gave disease-specific information $(77 \%$ vs $52 \%, P<0.01)$. Although PPC scores were equal in both groups, the average cost per patient of CGC follow-up was $25 \%$ lower. Follow-up of phenotype-negative relatives at risk for DCM/HCM at a CGC led to greater patient satisfaction and is well-appreciated at lower cost. CGC care is a good alternative to conventional cardiological follow-up for this growing group of patients.

European Journal of Human Genetics (2017) 25, 169-175; doi:10.1038/ejhg.2016.155; published online 30 November 2016

\section{INTRODUCTION}

Increasing cardiogenetic knowledge and availability of DNA analysis are leading to the identification of more phenotype-negative relatives at risk of developing an inherited cardiomyopathy. In accordance with the guidelines of the American College of Cardiology and the European Society of Cardiology, ${ }^{1,2}$ these relatives are advised to see a cardiologist for regular periodic monitoring. Important inheritable heart diseases include hypertrophic cardiomyopathy (HCM) and dilated cardiomyopathy (DCM): HCM has a familial background in $90 \%$ of cases, whereas DCM is familial in about $30 \%$ of cases. ${ }^{3}$ Thus, increasing numbers of asymptomatic relatives are qualifying for cardiological screening and follow-up every 2 years (for carriers of a variant that affects function) to 4 years (when no variant that affects function is identified in the index patient). ${ }^{1,2}$ This group of relatives includes carriers of a familial variant that probably or certainly affects function identified through cascade screening and first-degree relatives of symptomatic patients in whom no variant that affects function has, as of yet, been detected. This means that all at-risk close relatives are eligible for cardiologic screening and, depending on their age and screening results, for follow-up. Regular cardiological screening and follow-up are advised in these families to detect development of the disease early on, to alleviate morbidity and to prevent sudden cardiac death by providing treatment and lifestyle advice. What this means in practice is that greater numbers of phenotype-negative relatives at risk are taking up more of cardiologists' time, a resource that is already scarce and expensive.

The role of nurses, nurse practitioners and physician's assistants is of growing importance in the care of patients with cardiac diseases and those at risk. ${ }^{4-6}$ A study in low risk patients who had had a recent myocardial infarction demonstrated that nurse practitioners can deliver a quality of care equal to or better than conventional specialist care. ${ }^{4}$ Improved survival and self-care behaviour was seen in patients with heart failure when they were treated by specially educated and experienced cardiac nurses. ${ }^{4}$ The genetic counsellor in clinical genetics has a comparable role to these nurses and must, according to the European Board of Medical Genetics, be able to perform a range of tasks including providing information and facilitating the client's psychosocial adjustment to their genetic status and situation. ${ }^{7}$ Skirton et al. described how genetic counsellors have a significant workload associated with direct patient care and that this appears to be acceptable to patients. They further stated that the role of a genetic counsellor in specialist genetic settings could be adapted to integrate genetic counsellors into multidisciplinary teams in other specialties. ${ }^{7}$ Caleshu et al. ${ }^{8}$ conclude that 'cardiac genetic counsellors are ideally positioned to provide frontline psychological support for inherited cardiovascular disease families'. So far, a care model led by a genetic

\footnotetext{
${ }^{1}$ Department of Genetics, University Medical Center Groningen, University of Groningen, Groningen, The Netherlands; ${ }^{2}$ Department of Cardiology, University Medical Center Groningen, University of Groningen, Groningen, The Netherlands; ${ }^{3}$ Department of Cardiology, Antonius Hospital, Sneek, The Netherlands; ${ }^{4}$ Department of Clinical Genetics, Academic Medical Center, University of Amsterdam, Amsterdam, The Netherlands

${ }^{*}$ Correspondence: K Nieuwhof, Department of Genetics, University Medical Center Groningen, University of Groningen, PO Box 30001 , Groningen 9700 RB, The Netherlands. Tel: +31 50361 7229; Fax: +31 50361 7231; E-mail: k.nieuwhof@umcg.nl

Received 10 March 2016; revised 7 September 2016; accepted 13 October 2016; published online 30 November 2016
} 
counsellor/nurse for the follow-up of healthy relatives at risk of developing cardiomyopathy has not yet been evaluated, even though it may offer a straightforward solution to the problem of limited resources.

Our aim in this study was to compare the effectiveness of care in a cardiogenetic clinic (CGC) by an experienced genetic counsellor/nurse for relatives at risk of inherited cardiomyopathy with conventional care provided by cardiologists with respect to quality of cardiological care, satisfaction, perceived personal control (PPC) and costs. Our study hypothesis was that follow-up care provided by a trained genetic counsellor would be comparable with the conventional follow-up care provided by cardiologists.

\section{METHODS}

\section{Design and patients}

The study was designed as a randomised service evaluation of two different follow-up care modalities for first-degree relatives of index patients at risk for inherited cardiomyopathy, and was carried out between October 2011 and April 2013 in two Dutch hospitals: the University Medical Center Groningen (UMCG) and a regional hospital, the Antonius Hospital in Sneek (Trial registry ID ISRCTN35774505). The UMCG Medical Ethical Review Committee declared this study to be exempt from formal review and approval (M11.108973). Written informed consent was obtained from all participants. The service evaluation in this study was performed as an open label randomised comparison of two different follow-up care modalities.

First-degree relatives of index patients who were not already known to have a DCM/HCM diagnosis (based on their first screening at a combined Cardiogenetics Clinic), but who were eligible for follow-up according to information from the local patient database, were randomly assigned (1:1) at the beginning of the study and stratified by one of the hospitals to one of our two care models:

(i) A CGC with a genetic counsellor with a nursing background, who was trained in cardiogenetics and follow-up care, supervised by a cardiologist.

(ii) Regular follow-up care by a cardiologist.

Both care models were provided in both hospitals. Professionals involved in the care models were not blinded to the randomisation result. Care models were randomised to individuals. For individuals who preferred to have followup care with a close relative $(n=5)$, both were assigned together to either (i) or (ii).

Inclusion criteria for participants were:

- They were either phenotype-negative relatives (over 16 years of age) of index patients with DCM or HCM with a proven variant that affects function and therefore at risk for developing DCM or HCM.

- They were carriers of variants that affect function in the LMNA, DES or PLN genes, who are at a higher prior risk for malignant ventricular arrhythmias compared with the other groups. ${ }^{9,10}$

- They were phenotype-negative relatives of index patients with potentially inherited DCM or HCM in whom no variant that affects function had been identified.

- All participants had been counselled at the Department of Genetics, UMCG. Counselling included discussing family health status, providing information about the disease and its (potential/possible) inheritance, discussing the consequences for pregnancies and (future) children, discussing (dis)advantages and consequences of DNA- and cardiologic investigations and clarifying the (possible) increased risk of getting the inherited disease.

Exclusion criteria were:

- Any signs or symptoms of the disease.

- The presence of other heart diseases.

- A medical history with complex co-morbidity.

- Non-Dutch speaking.

\section{Modes of follow-up care}

The specialist clinic was led by cardiologists (with initials MPvdB, RAdB, $\mathrm{PLvH}$ ) with experience in diagnosing and managing cardiogenetic diseases. In this conventional approach, relatives had to make their own appointment for follow-up if the next visit was planned for more than one year later; otherwise the next appointment was made during the current visit. Cardiological care usually consisted of an electrocardiogram (ECG), echocardiography, measurement of blood pressure, assessment of the patient's health and occasionally sharing of information about DCM/ HCM or discussing the option to reconsider predictive DNA testing and/ or to recontact the Department of Genetics (eg, if considering a pregnancy). The time scheduled for such a consultation was $15 \mathrm{~min}$ (covering the preparation for the consultation, the consultation itself and reporting findings to the general practitioner).

The CGC was led by a trained genetic counsellor (with initials $\mathrm{KN}$ ), a registered nurse with a Master of Science in Nursing Science, who was educated to become a genetic counsellor in the Netherlands according to the national training programme acknowledged by the Dutch Clinical genetics Association (VKGN). Because there is no specific Master's programme to train genetic counsellors in the Netherlands and in some other European countries, a grandfather clause was introduced by the European Board of Medical Genetics for genetic counsellors working in these countries. Under this clause, genetic counsellors can register by completing a portfolio that demonstrates they have all the required education and competencies (https://www.eshg.org/471.0.html). The genetic counsellor in our study had worked as a nurse in the Department of Cardiology for several years and who had performed cardiogenetic counselling and testing at the Department of Genetics for over ten years. The counsellor's knowledge and skills were optimised by following an ECG course and by practical training in interpreting images and descriptions of outcomes of echocardiography. Specific protocols for following-up relatives at risk for DCM, HCM and specific gene-related disorders, as well as pregnant women at risk of developing an inherited cardiomyopathy, were developed by the genetic counsellor in collaboration with the cardiologists and we can expect that both professions were equally knowledgeable about their contents. These protocols were based on national and international guidelines ${ }^{1,2}$ and on the practical experience of cardiologists with this population, and were applied in both models of care. The genetic counsellor performed follow-up under the supervision of cardiologists with experience in cardiogenetic diseases (MPvdB, RAdB, PLvH). We considered these factors to be of major importance in starting a CGC.

The CGC-approach consisted of sending an invitation to eligible relatives at risk with a request to schedule an appointment for follow-up. The care consisted of an ECG, echocardiography, measurement of blood pressure, assessment of the patient and his/her family's health and, as needed, providing information on DCM/HCM. The genetic counsellor also asked about any new developments in the family, such as relatives who may have developed the disease or had died suddenly. In addition, she discussed reproductive options when appropriate and discussed new diagnostic/ treatment options or the results of DNA testing. If applicable, she urged participants to encourage other relatives to have genetic or cardiological screening. If the participants consented to predictive DNA testing, the genetic counsellor initiated this procedure immediately after the counselling session. Guided by the results of the cardiological investigations, according to the protocols and/or the patient/family history, the counsellor could propose further tests such as a Holter monitor, an exercise stress test or a cardiac MRI. This policy was discussed immediately (in another room) with the supervising cardiologist (multidisciplinary context) and, in a joint consultation, additional diagnostics were discussed with the patient. The cardiologist later discussed the results of additional diagnostic tests with the participant. The patient investigations of all patients were reviewed by the cardiologist. The supervising cardiologist oversaw the report of findings in a letter and took final responsibility for the care provided. The time scheduled for a consultation (covering the preparation, the consultation including discussing the outcomes of investigation with the patient, the supervision by the cardiologist and reporting findings to the general 
practitioner) was $25 \mathrm{~min}$. This time length was a try-out and contained more scheduled time because of the cardiologic supervision of the CGC during the consultation (this was not necessary at the specialist clinic). This supervision time after echo counselling was scheduled to guarantee the patient received the correct test results before going home. The actual time taken for a consultation at the CGC was always recorded.

Both types of caregivers had access to the same facilities in the outpatient clinic in both hospitals: a room, a computer and secretarial assistance.

\section{Endpoints}

The primary outcome measure was the average proportion of follow-up care provided. That proportion was defined as the average proportion of the evaluation of patient health, health status of the family, blood pressure, giving of information, ECG and echocardiogram. The other endpoints were defined as secondary outcomes: the uptake percentage of follow-up by relatives at either clinic during the study; PPC and patient satisfaction, which are both accepted outcome measures for patient-related outcomes of care; results of supervision, resource use and cost reductions. The uptake rates at both clinics were calculated as the percentage of relatives who actually attended the clinic compared with the total number eligible for follow-up (based on local patient databases) during this study.

\section{Instruments}

Participants received a survey within 2 weeks after the follow-up visit. Participants at the CGC received the questionnaire from the CGC directly after the follow-up visit. Participants at the cardiologist' clinic received the survey at one location from the cardiologist and at the other location from the outpatient secretary, sometimes not directly but within 2 weeks after the consultation. Both the genetic counsellor and cardiologist were informed about the questions in the questionnaire and aware of the endpoints measured, and they introduced the questionnaire only briefly by saying that they wanted to know how the patient experienced the visit. The survey contained questions about PPC, satisfaction and the care process. Participants' PPC was measured using the Dutch validated PPC questionnaire. ${ }^{11,12}$ The validated nine-item PPC (Dutch version) was used to measure a patient's perceived control before and after genetic counselling. The response mode is a $0-2$ Likert scale. Higher scores indicate higher levels of perceived control (0 means no control and 2 means full control).

Patient satisfaction was evaluated with the Satisfaction Indicator of the Dutch Clinical Genetics Association (VKGN), and is part of the quality criteria of the VKGN. This seven-item questionnaire was adopted by the Clinical Genetics Association in the Netherlands to measure patient satisfaction with genetic counselling. It is the Dutch version of the UK questionnaire developed by Zellerino et al. ${ }^{13}$ The response mode is a 1-5 Likert scale. Higher scores indicate higher satisfaction ( 1 means no satisfaction and 5 means maximum satisfaction). The survey also contained several statements on the care process and what care was given, for example:

- The healthcare provider inquired about my health status.

- The healthcare provider inquired about the health status of family members.

- Oral information about the cardiomyopathy and its inheritance was provided.

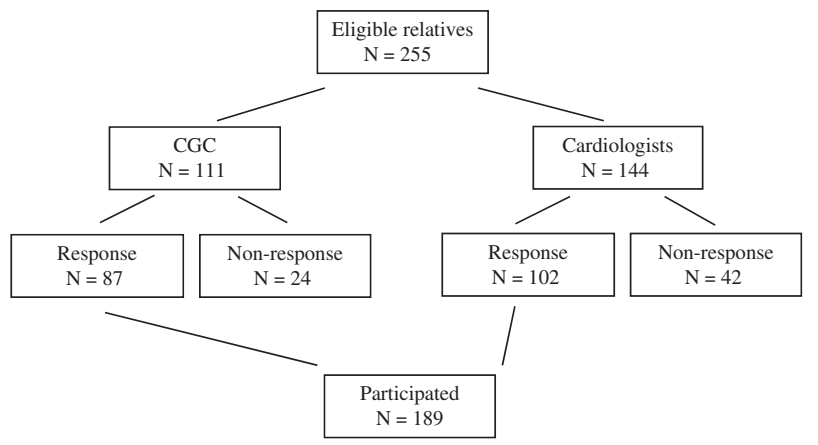

Figure 1 Flow of relatives/participants.
We selected both the PPC questionnaire as well as a patient satisfaction questionnaire because we were interested in relatives' experiences of control and the care process. The PPC essentially focusses on the effectiveness of the visit in terms of control, whereas the Satisfaction Indicator examines a patient's experience with the provision of care. The outcomes and resource use of those who visited the cardiologist's clinic were collected and analysed from their medical correspondence.

\section{Cost calculations}

The cost consequences of each mode of follow-up care were expressed as the average costs per patient from the hospital perspective. Hospital costs included the costs of developing a CGC-based follow-up protocol and ECG and echocardiography training (CGC only), monitoring which relatives were eligible for follow-up (CGC only), sending out invitations and reminders, planning the consultations, preparing for the consultations and performing follow-up consultations including supervision by the cardiologists (CGC only). Costs beyond supervision were disregarded because supervision showed that the genetic counsellor's consultations and her referrals were judged correct in all cases. Costs were calculated as professionals' time spent on each phase of the care process multiplied by their gross hourly wage rates (including taxes and social premiums, excluding departmental and hospital overheads; at the end of 2015 price level). The costs of protocol development and training were estimated as the total costs of the professionals amortised over an (assumed) 10-year economic life span. A one-way sensitivity analysis was added to check the degree to which the estimated cost difference between modes of follow-up care was vulnerable to the projected times spent and wage rates.

\section{Statistics}

At study onset, we had no prior information to serve as basis for a formal sample size calculation.

Data were analysed according to intention to treat. Differences in uptake rates and the proportions of top-ratings of satisfaction and resource use were compared in IBM SPSS Statistics software 22.0 (IBM, Armonk, NY, USA) using the $\chi^{2}$ test. PPC scores were reported as median (interquartile range (IQR)) scores and tested between the two follow-up modalities using the nonparametric Mann-Whitney $U$ test. Proportions of maximum score per PPC item were compared using Fisher's exact test. Differences in mean age were compared using the Mann-Whitney $U$ test. Differences in outpatient time between the CGC and the standard 15-min cardiologist follow-up was compared using the one sample Student's $t$-test. A $P$-value $<0.05$ (two-sided) was considered to be statistically significantly different.

\section{RESULTS}

\section{Population and uptake}

Figure 1 depicts the flow of relatives/participants. Of 255 eligible relatives, $189(74 \%)$ participated in the study and visited one of our clinics. Their age range was $16-78$ years and $46 \%$ were men. Of the 189 relatives, $50 \%$ were proven carriers of a variant that affects function. The remainder had a positive family history of DCM/HCM (but without an identified variant that affects function in the index patient) or had chosen not to undergo predictive testing for a familial variant that affects function. In total, 87 out of 111 relatives $(79 \%)$ who were invited for follow-up visited the CGC vs 102 out of 144 (71\%) who saw the cardiologist (Figure 1). The difference in the number of relatives invited for follow-up between the two clinics reflects a large number of relatives who visited the cardiologist's clinic with a close relative. The uptake rates at both clinics were not significantly different $(P=0.17)$.

Table 1 shows the characteristics of the relatives who visited a clinic for follow-up (responders) and those who did not (non-responders). There were no significant differences in characteristics between responders visiting the CGC or the cardiologist's clinic. 
Table 1 Characteristics of responders and non-responders

\begin{tabular}{|c|c|c|c|c|}
\hline Characteristics & Genetic counsellor & Cardiologist & & Total \\
\hline Responders & $N=87$ & $\mathrm{~N}=102$ & P-value & $N=189$ \\
\hline Men & $41(47 \%)$ & $45(44 \%)$ & 0.62 & $86(46 \%)$ \\
\hline Carriership & & & 0.73 & \\
\hline Carrier & $41(47 \%)$ & $54(53 \%)$ & & $95(50 \%)$ \\
\hline Uncertain carrier & $7(8 \%)$ & $8(7 \%)$ & & $15(8 \%)$ \\
\hline PLN & $14(16 \%)$ & $19(19 \%)$ & & $33(17 \%)$ \\
\hline DCM & 30 (34\%) & 40 (39\%) & & $70(37 \%)$ \\
\hline Desminopathy & 0 & $3(3 \%)$ & & $3(2 \%)$ \\
\hline $\mathrm{HCM}$ & $43(49 \%)$ & 40 (39\%) & & $83(44 \%)$ \\
\hline Non-responders & $N=24$ & $N=42$ & & $N=66$ \\
\hline Uncertain carrier & 0 & 0 & & $0(0 \%)$ \\
\hline No variant found in family & $22(92 \%)$ & $37(88 \%)$ & & $59(89 \%)$ \\
\hline Disease/gene & & & 0.37 & \\
\hline$P L N$ & 0 & $1(2 \%)$ & & $1(2 \%)$ \\
\hline DCM & $21(88 \%)$ & $29(69 \%)$ & & $50(76 \%)$ \\
\hline Desminopathy & 0 & 0 & & $0(0 \%)$ \\
\hline $\mathrm{HCM}$ & $3(12 \%)$ & $12(29 \%)$ & & $15(23 \%)$ \\
\hline
\end{tabular}

Abbreviations: DCM, dilated cardiomyopathy; HCM, hypertrophic cardiomyopathy; PLN, phospholamban.

Table 2 Perceived personal control with median overall scores and maximum scores per item for both clinics

\begin{tabular}{|c|c|c|c|}
\hline & $\begin{array}{l}\text { Genetic counsellor } \\
\qquad \mathrm{N}=83^{\mathrm{a}}\end{array}$ & $\begin{array}{l}\text { Cardiologist } \\
\qquad \mathrm{N}=72^{\mathrm{a}}\end{array}$ & P-value \\
\hline PPC score (median, IQR) & $1.33(0.78-1.56)$ & $1.22(1.00-1.67)$ & 0.39 \\
\hline I think I understand the problem that brought me here & $76(92 \%)$ & $67(93 \%)$ & 0.77 \\
\hline I feel I know the meaning of the problem for my family's future and for me & $56(68 \%)$ & $48(68 \%)$ & 0.99 \\
\hline I think I know what caused the problem & $42(51 \%)$ & $44(63 \%)$ & 0.14 \\
\hline I feel I have the tools to make decisions that will influence my future & $41(49 \%)$ & $38(54 \%)$ & 0.63 \\
\hline I feel I can make a logical evaluation of the various options available to me in order to choose one of them & $37(45 \%)$ & $34(49 \%)$ & 0.63 \\
\hline I feel I can make decisions that will change my family's future & $25(30 \%)$ & $26(37 \%)$ & 0.49 \\
\hline I feel there are certain things I can do to prevent the problem from re-occurring & $17(21 \%)$ & $15(21 \%)$ & 0.99 \\
\hline I feel I know what to do to ease the situation & $16(19 \%)$ & $20(29 \%)$ & 0.19 \\
\hline I think I know what my next steps should be & $27(33 \%)$ & $26(37 \%)$ & 0.81 \\
\hline
\end{tabular}

Abbreviation: PPC, perceived personal control.

a Numbers of patients for each PPC item may vary due to missing values (nurse: 82-83; cardiologist: 69-72).

\section{Perceived personal control}

Table 2 displays the PPC results. Of 87 relatives who visited the CGC, 83 (95\%) filled in the questionnaire about PPC vs 72/102 (71\%) relatives who visited the cardiologist's clinic. Median (IQR) PPC between both clinics was comparable: 1.33 (IQR: 0.78-1.56) for the CGC vs 1.22 (IQR: $1-1.67)$ for the cardiologist's clinic $(P=0.39)$.

\section{Patient satisfaction}

Of the 102 relatives who visited the cardiologist's clinic, 73 (72\%) returned the patient satisfaction questionnaire. All those who visited the CGC returned the questionnaire (100\%).

The maximum patient satisfaction score was seen more often at the CGC than at the cardiologist's clinic ( $86 \%$ vs $45 \%, P<0.01)$. Participants seen at the CGC reported more often that the caregiver listened to them ( $97 \%$ vs $84 \%, P=0.02)$, that they felt understood by the caregiver ( $95 \%$ vs $78 \%, P<0.01$ ), that the caregiver provided good information $(93 \%$ vs $81 \%, P=0.03)$ and that they felt their opinion was appreciated by the care provider $(91 \%$ vs $59 \%, P<0.01)$. At the CGC, participants much appreciated the caregiver's time ( $94 \%$ vs $77 \%$, $P=0.01$ ), as well as being given the opportunity to exchange information (94\% vs 79\%, $P=0.02$ ) and the manner in which the caregiver answered their questions (94\% vs 81\%, $P=0.01$ ) (Table 3).

\section{Efficiency, resource use and costs}

The genetic counsellor consulted the cardiologist for certain cases such as cardiac health complaints or abnormal diagnostic results as prescribed in the dedicated protocols. 
Table 3 Patient satisfaction score

\begin{tabular}{|c|c|c|c|}
\hline Patient satisfaction items & $\begin{array}{l}\text { Genetic counsellor } \\
\qquad \mathrm{N}=87\end{array}$ & $\begin{array}{l}\text { Cardiologist } \\
\qquad \mathrm{N}=73\end{array}$ & $\mathrm{P}$-value \\
\hline Max patient satisfaction score & $75(86 \%)$ & $33(45 \%)$ & $<0.01 *$ \\
\hline \multicolumn{4}{|l|}{ Caregiver } \\
\hline Listens to the patient & $84(97 \%)$ & $61(84 \%)$ & $0.02 *$ \\
\hline Understands the patient & $83(95 \%)$ & $57(78 \%)$ & $<0.01^{*}$ \\
\hline Provides good information & 81 (93\%) & $59(81 \%)$ & $0.03^{*}$ \\
\hline Appreciates patient's opinion & 79 (91\%) & $43(59 \%)$ & $<0.01^{*}$ \\
\hline Takes enough time for the patient & 82 (94\%) & $56(77 \%)$ & $0.01 *$ \\
\hline $\begin{array}{l}\text { Provides opportunity to exchange } \\
\text { information }\end{array}$ & $82(94 \%)$ & $58(79 \%)$ & $0.02 *$ \\
\hline Answers questions of the patient & $82(94 \%)$ & $59(81 \%)$ & $0.01 *$ \\
\hline
\end{tabular}

Table 4 Follow-up care processes at the cardiology and nurse-led clinics

\begin{tabular}{|c|c|c|c|}
\hline Care process & $\begin{array}{c}\text { Genetic } \\
\text { counsellor } \\
\mathrm{N}=87\end{array}$ & $\begin{array}{l}\text { Cardiologist } \\
\qquad \mathrm{N}=102\end{array}$ & P-value \\
\hline Evaluation of patient's health & $87(100 \%)$ & $66(65 \%)$ & $<0.01^{*}$ \\
\hline Family's health status & $84(97 \%)$ & $34(33 \%)$ & $<0.01$ * \\
\hline Blood pressure & $85(98 \%)$ & $30(29 \%)$ & $<0.01$ * \\
\hline Giving information & $67(77 \%)$ & $53(52 \%)$ & $<0.01 *$ \\
\hline Electrocardiogram & $86(99 \%)$ & 92 (90\%) & $0.04^{*}$ \\
\hline Echocardiogram & $86(99 \%)$ & $89(87 \%)$ & $0.01 *$ \\
\hline Extra diagnostics & $27(31 \%)$ & $27(26 \%)$ & 0.15 \\
\hline $\begin{array}{l}\text { Referral to cardiologist by genetic counsellor } \\
\text { for phenotypic signs of cardiomyopathy }\end{array}$ & $6(7 \%)$ & 0 & \\
\hline Average proportion of follow-up care provided & $95.0 \%$ & $59.3 \%$ & $<0.01$ \\
\hline
\end{tabular}

${ }^{*} P<0.05$ (statistically significant).

Compared with the cardiologist's clinic, relatives attending the CGC more often reported their health status taken $(100 \%$ vs $65 \%, P<0.01)$, more often had their family health status evaluated ( $97 \%$ vs $33 \%$, $P<0.01)$ and more often had their blood pressure measured $(98 \%$ vs $29 \%, P<0.01)$. They also reported more often being given information about the disease and its inheritance in the family (77\% vs 52\%, $P<0.01$ ) (Table 4). The average proportion of follow-up care provided (evaluation of patient's health, family's health status, blood pressure, giving information, ECG and echocardiogram) was 95\% for the genetic counsellor and $59.3 \%$ for the cardiologist $(P<0.01)$, indicating that the genetic counsellor did not perform worse than the cardiologist in this respect.

Extra diagnostic tests were requested, if indicated, in about equal proportions by the genetic counsellor (after consulting the cardiologist) and by the cardiologists (Table 4).

The mean duration of a consultation at the CGC (mean 15 min, range $13-17 \mathrm{~min}$ ) did not differ significantly from the standard length of time scheduled for a consultation with the cardiologist (15 min). However, the average costs per patient were 25\% lower in the CGC-based follow-up than in conventional follow-up by cardiologists ( $€ 26.39$ vs $€ 35.22$; Tables 5 and 6). In both modes of care, $75-80 \%$ of the costs of care were spent in the actual followup visit and subsequent supervision $(73.1 \%$ for the CGC model $v s$
Table 5 Average costs per mode of follow-up care

\begin{tabular}{lcc}
\hline & $\begin{array}{c}\text { Genetic counsellor } \\
\text { Costs }(€)\end{array}$ & $\begin{array}{c}\text { Cardiologist } \\
\text { Costs }(€)\end{array}$ \\
\hline Protocol development and training & 2.58 & 0 \\
Invitation, booking, rescheduling appointment & 3.14 & 2.39 \\
Follow-up visit—-preparation & 1.38 & 3.86 \\
Follow-up visit-actual visit and administration & 10.38 & 28.96 \\
Supervision, evaluation & 8.92 & 0 \\
Average costs per patient & 26.39 & 35.21 \\
\hline
\end{tabular}

$82.2 \%$ for follow-up by the cardiologist). If supervision by the cardiologist is not necessary, the cost difference rises to $50.4 \%$ in favour of CGC care. If supervision by the cardiologist is limited to the cases in which cardiologist consultation is indicated ( 6 out of 87 cases), the cost difference would be $48.6 \%$ in favour of CGC care.

The sensitivity analyses revealed that the estimated cost difference was relatively sensitive to the duration of the follow-up visit of the cardiologist and cardiologist's wage rate (Table 6). An increase in the duration of the genetic counsellor's follow-up visit $(+20 \%)$ or the genetic counsellor's wage rate $(+10 \%)$ would still produce a cost reduction $(-19.2 \%$ and $-20 \%$, respectively, instead of $-25.1 \%)$ in favour of the CGC mode of care.

\section{DISCUSSION}

We have compared two different care modalities to provide cardiogenetic follow-up for healthy relatives at risk of developing an inherited cardiomyopathy: conventional care by a cardiologist $v s$ care provided in CGC led by a genetic counsellor and supervised by the cardiologist. Such a substitution of care has not been reported before in the setting of inherited cardiomyopathy. Our study shows that relatives who attended the CGC experienced equally good or better care (conforming with protocols and with justified supervision) and were satisfied with the care provided, whereas the cost of the CGC was lower than that of a cardiologist's clinic. Our findings are relevant for health authorities organising care for the growing group of relatives who have been identified as at risk for cardiomyopathy, and who need routine follow-up, at a time when available health resources are already under increasing demand.

Previous studies in cardiac care have reported on the effects of partially replacing specialist care with nurse care, but comparisons with our study are difficult to make. First, in our study, most participants saw one only caregiver, whereas in previous studies examining the role of nurses in heart failure clinics, the patient was always seen by various caregivers, including a cardiologist. ${ }^{4-6}$ Second, our study group consisted of symptom-free relatives, whereas previous studies covered patients diagnosed with a cardiac or other disease. Finally, the setting of our study differed from that of previous studies. In our study, the genetic counsellor is a healthcare provider who functions as a liaison between the Departments of Genetics and Cardiology (at both study sites): first in the counselling process, often with predictive genotyping, at the Department of Genetics, and then in cardiological follow-up at the Department of Cardiology. In previous studies, only one department and type of care process were involved. Patients probably appreciated having a single individual with knowledge and skills in both Cardiology and Genetics, which was not the case in the other studies.

Despite the differences with previous studies, our main findingthat cardiological care could be managed adequately by an experienced 
genetic counsellor (who is also a qualified nurse) at lower costs-is in agreement with previous studies on cardiological care provided by a nurse. ${ }^{4-6}$

It must be mentioned that genetic counsellors worldwide do not always have the same education. Although the genetic counsellor training programme in the Netherlands is organised differently compared witth those of other European nations, the competencies and skills acquired are similar to other European programmes. Because, as in some other European countries, Dutch genetic counsellor training is not a Master's programme, a specific grandfather clause was established by the European Board of Medical Genetics Professional Branch for genetic nurses and counsellors working in countries with no current system of registration (https://www.eshg.org/471.0.html). Under this clause, genetic counsellors can register by completing a portfolio, which contains work experience in genetic counselling, case logs, case studies, references, courses (or examinations) and continuing professional development records (see https://www.eshg.org/471.0.html for details of the registration process). Courses are expected to be at postgraduate level and must be validated by the host institution. By introducing the Grandfather clause the difference in education of genetic counsellors is being equalised. We therefore expect that more genetic counsellors with a nursing background and with further education in interpreting test results of cardiologic examination ('training on the job') can offer this service.

The strengths of our study lie in our comparison of two follow-up modes in a randomised fashion in an unselected group of relatives and in the successful implementation of this new care mode in two hospitals. Moreover, the estimated costs and sensitivity analysis suggest that the cost reduction of CGC-based follow-up care is a robust result. Furthermore, we used a comparative approach through a randomised comparison of two follow-up care models, and took conventional follow-up care by cardiologists as comparator. We feel this choice is a strength of our approach.

However, there are some possible limitations to this study. First, we could not calculate a sample size before study onset. Given the 189 relatives included and the average proportion of follow-up care provided (95\% for genetic counsellor/nurse and $59.33 \%$ for the cardiologist; Table 4), we could demonstrate that the genetic counsellor/nurse did not perform worse than the cardiologist with a power of over $95 \%$, providing justification for the validity of our study from a non-inferiority perspective. Some researchers have criticised the non-inferiority approach for two reasons. One is that noninferiority allows the study power to depend on the selected noninferiority margin (ie, the range with which the trained $\mathrm{GC} /$ nurse is allowed to perform 'minimally' worse than the cardiologist and still be regarded as about comparable to the cardiologist). Non-inferiority tends to reward the careless, ${ }^{14}$ as the statistical test result may partially depend on the optimality of cardiologists' performance. Although not our aim, it should be noted that the alternative approach of superiority testing would produce a post-hoc power of $>99 \%$. Second, we cannot exclude that the caregiver's sex may have influenced patient satisfaction or PPC results as the genetic counsellor was female whereas the cardiologists were male. ${ }^{15}$ Third, the comparison of the care processes was evaluated partly by questionnaires; we cannot exclude that the use of patient-reported measures may have introduced selective nonresponse, misinterpretation and/or response tendencies. Thus it is possible that some questions were misunderstood by patients filling in the questionnaires, making their answers about the care process less reliable. It is also conceivable that sending an invitation to the patients to visit the CGC positively influenced the patient satisfaction. Fourth, differences in the care received in the cardiologist's clinic may be due to the cardiologist not strictly following international guidelines and/or protocols. After years of experience, they may decide to deviate from the protocol with proper motivations or arguments, which is allowed as long as their motivation is documented in the patient files. Further assessment of why these protocol deviations occurred was not part of our study. Fifth, the fact that there are a considerable number of nonresponders could be assigned to a CGC, that is, non-respondents were driven away by the prospect of being seen in the CGC. We think this is highly unlikely because the non-response at the CGC follow-up clinic was actually lower than non-response at the cardiologist's follow-up clinic $(21.6 \%$ vs $29.2 \%$, respectively) and because the reasons for non-response were mainly related to having relatives who consulted a cardiologist in a nearby hospital or for to the financial burden of the health insurance co-payment. Finally, our study focussed on the substitution of care between professionals. Differences in long term health outcomes are unlikely to originate from differences in caregiver at the follow-up clinic because the genetic counsellor's consultations and referrals were correct in all cases.

We recommend that further studies should be conducted to evaluate the transition of care from cardiologists to genetic counsellors with a nursing background in three aspects. First, to investigate if the CGC can also be implemented successfully in other, perhaps smaller and less specialised, hospitals and in different healthcare systems and other countries. We would expect specialised cardiogenetic counsellors in other settings to be able to offer such follow-up care as long as their knowledge and skills are sufficient and the organisation is satisfactory. 
Second, the joint nursing background and counselling background seem to be important in this care setting because the genetic counsellor's function at the CGC combines medical judgement and genetic counselling skills. Nurses, nurse practitioners or physician assistants who do not have the genetic counselling background may lack these competencies and may not be qualified to offer the same care as a genetic counsellor can offer at a CGC. In Europe, most genetic counsellors have a nursing background, and it is possible that those without are not competent in making medical judgements. Competencies need to be described systematically and subsequently investigated in potential candidates to guarantee proper care. It needs to be investigated whether the CGC can be equally successful when led by cardiac nurses, nurse practitioners or physician assistants trained in genetic counselling, or by genetic counsellors without a nursing background but with training in this kind of cardiological follow-up care. Third, further studies should investigate if the same model of care can also be successfully applied to different inherited cardiac or cardiovascular patient groups such as phenotype-negative relatives at risk for aortic diseases like Marfan syndrome or familial thoracic aortic aneurysms and dissections.

We conclude that genetic counsellors with extra training in cardiac diseases can provide satisfactory follow-up for relatives at risk for cardiomyopathy if these counsellors work according to protocols and under the supervision of a cardiologist experienced in cardiogenetics. These counsellors can provide at least a similar quality of cardiological care to that offered by cardiologists, but with increased patient satisfaction and at lower cost. A CGC should therefore be considered a feasible care modality to standard follow-up provided by cardiologists.

\section{CONFLICT OF INTEREST}

The authors declare no conflict of interest.

\section{ACKNOWLEDGEMENTS}

We thank all the participants and the Department of Cardiology of the University Medical Center Groningen (UMCG) and the Antonius Hospital in Sneek. We also thank Jackie Senior and Kate Mc Intyre for editing this manuscript. This work was supported by the Health Care Efficiency Research
Pilot programme of the University Medical Center Groningen, Groningen, the Netherlands.

1 Maron BJ, McKenna WJ, Danielson GK et al: American College of Cardiology/European Society of Cardiology Clinical Expert Consensus Document on Hypertrophic Cardiomyopathy; a report of the American College of Cardiology Foundation Task Force on Clinical Expert Consensus Documents and the European Society of Cardiology Committee for Practice. Eur Heart J 2003; 24: 1965-1991.

2 Gersh BJ, Maron BJ, Bonow RO et al: 2011 ACCF/AHA Guideline for the Diagnosis and Treatment of Hypertrophic Cardiomyopathy: a report of the American College of Cardiology Foundation/American Heart Association Task Force on Practice Guidelines. J Am Coll Cardiol 2011; 58: e212-e260.

3 Watkins H, Ashrafian BM, Redwood C: Inherited cardiomyopathies. N Engl J Med 2011; 364: 1643-1656.

4 Strömberg A, Mårtensson J, Fridlund B et al: Nurse-led heart failure clinics improve survival and self-care behaviour in patients with heart failure. Results from a prospective, randomised trial. Eur Heart J 2003; 24: 1014-1023.

5 Broers CJM, Sinclair N, Van der Ploeg TJ et al: The post-infarction nurse practitioner: a prospective study comparing nurse intervention with conventional care in a non-highrisk myocardial infarction population. Neth Heart J 2009; 17: 61-67.

6 Jaarsma T, Strömberg A: Heart failure clinics are still useful (more than ever?). Can J Cardiol 2014; 30: 272-275.

7 Skirton H, Cordier C, Ingvoldstad C et al: The role of the genetic counsellor: a systematic review of research evidence. Eur J Hum Genet 2015; 23: 452-458.

8 Caleshu C, Kasparian NA, Edwards KS et al: Interdisciplinary psychosocical care for families with inherited cardiovascular diseases. Trends Cardiovasc Med 2016; 26: 647-653.

9 Van Spaendonck-Zwarts KY, Van Rijsingen IA, Van den Berg MP et al: Genetic analysis in 418 index patients with idiopathic dilated cardiomyopathy: overview of 10 years' experience. Eur J Heart Fail 2013; 15: 628-636.

10 Van der Zwaag PA, Van Rijsingen IA, Asimaki A et al: Phospholamban R14del in patients diagnosed with dilated cardiomyopathy or arrhythmogenic right ventricular cardiomyopathy: evidence supporting the concept of arrhythmogenic cardiomyopathy. Eur J Heart Fail 2012; 14: 1199-1207.

11 Berkenstadt M, Shiloh S, Barkai G et al: Perceived personal control (PPC): a new concept in measuring outcome of genetic counseling. Am J Med Genet 1999; 82. 53-59.

12 Smets EMA, Pieterse $\mathrm{AH}$, Aalfs $\mathrm{CM}$ et al: The perceived personal control (PPC) questionnaire as an outcome of genetic counseling: reliability and validity of the instrument. Am J Med Genet 2006; 140: 843-850.

13 Zellerino B, Milligan SA, Brooks R et al: Development, testing, and validation of a patient satisfaction questionnaire for use in the clinical genetics setting. Am J Med Genet 2009; 151C: 191-199.

14 Schumi J, Witte JT: Through the looking glass: understanding non-inferiority. Trials 2011; 12: 106

15 Bertakis KD, Franks P, Azari R: Effects of physician gender on patient satisfaction. J Am Womens Assoc 2003; 58: 69-75. 